Research Paper

\title{
Evaluation of chromogenic media and seminested PCR in the identification of Candida species
}

\author{
Enas Daef ${ }^{1}$, Ahmed Moharram $^{2}$, Salwa Seif Eldin ${ }^{1}$, Nahla Elsherbiny ${ }^{1}$, \\ Mona Mohammed ${ }^{1}$ \\ ${ }^{1}$ Medical Microbiology and Immunology Department, Faculty of Medicine, Assiut University, \\ Assiut, Egypt. \\ ${ }^{2}$ Botany Department, Faculty of Science, Assiut University, Assiut, Egypt.
}

Submitted: June 25, 2012; Approved: April 1, 2013.

\begin{abstract}
Identification of Candida cultured from various clinical specimens to the species level is increasingly necessary for clinical laboratories. Although sn PCR identifies the species within hours but its cost-effectiveness is to be considered. So there is always a need for media which help in the isolation and identification at the species level. The study aimed to evaluate the performance of different chromogenic media and to compare the effectiveness of the traditional phenotypic methods $v s$. seminested polymerase chain reaction (sn PCR) for identification of Candida species. One hundred and twenty seven Candida strains isolated from various clinical specimens were identified by conventional methods, four different chromogenic media and sn PCR. HiCrome Candida Differential and CHROMagar Candida media showed comparably high sensitivities and specificities in the identification of C. albicans, C. tropicalis, C. glabrata and C. krusei. CHROMagar Candida had an extra advantage of identifying all $C$. parapsilosis isolates. CHROMagar-Pal's medium identified $C$. albicans, C. tropicalis and C. krusei with high sensitivities and specificities, but couldn't identify $C$. glabrata or C. parapsilosis. It was the only medium that identified C. dubliniensis with a sensitivity and specificity of $100 \%$. Biggy agar showed the least sensitivities and specificities. The overall concordance of the snPCR compared to the conventional tests including CHROMAgar Candida in the identification of Candida species was $97.5 \%$. The use of CHROMAgar Candida medium is an easy and accurate method for presumptive identification of the most commonly encountered Candida spp.
\end{abstract}

Key words: chromogenic media, sn PCR, Candida.

\section{Introduction}

Invasive candidiasis is recognized as a leading cause of morbidity and mortality in both immunocompetent and immunocompromised critically ill patients. In intensive care unit (ICU) patients, the most common types of Candida infections are bloodstream, intra-abdominal, urinary tract and catheter-related infections. Until recently, Candida albicans was by far the predominant species, causing up to two-thirds of all cases of invasive candidiasis. However, a shift toward non-albicans Candida spp. (NAC) with reduced susceptibility to commonly used antifungal agents, was recently observed (Nguyen et al., 1996).
Changing candidal epidemiology and the availability of newer antifungal drugs with different antifungal spectrums means that physicians can no longer make therapeutic decisions based on the broad identification of fungi as either yeasts or moulds (Hospenthal et al., 2004). Prompt diagnosis of infection is of utmost importance as rapid initiation of appropriate antifungal therapy is crucial for reducing mortality (Fluit et al., 2000).

Identification to the species level of yeasts cultured from various clinical specimens is increasingly necessary for clinical laboratories. Nowadays, a large variety of Candida spp. identification methods are commercially available, and they differ in principles, discrimination 
power and cost. Generally yeast identification procedures start with a germ tube test in clinical laboratories. When the yeast cannot be identified with this method, further tests such as culturing on cornmeal agar, carbohydrate fermentation and carbohydrate assimilation tests are performed. Detection of growth patterns on cornmeal agar takes 24-72 h and sugar assimilation tests may take $72 \mathrm{~h}$ to two weeks. These procedures are labor intensive and take longer to determine the diagnosis and judge the proper antifungal agent (Baradkar et al., 2010).

In order to facilitate rapid identification, several chromogenic substrate containing culture media have been developed. These special media yield microbial colonies with varying colors secondary to chromogenic substrates that react with enzymes secreted by microorganisms (Bernal et al., 1996). Chromogenic agar carries the potential of improving identification of yeast, especially in mixed cultures (Murray et al., 2005).

In order to overcome the limitations of conventional diagnostic tests, DNA-based methods have been developed for the detection of Candida species and offer a potentially more sensitive means of identification of the yeasts and they appear reliable and easy to use (Liguori et al., 2007). DNA amplification with universal fungal primers followed by detection using species-specific probes greatly improved the sensitivity of Candida detection (Wahyuningsih et al., 2000). This study aimed to evaluate the performance of four different chromogenic media and to compare the effectiveness and practical applicability of the traditional phenotypic methods vs. sn PCR for identification of Candida to the species level.

\section{Material and Method}

\section{Clinical isolates}

One hundred and twenty seven Candida strains were isolated from different clinical specimens from patients with nosocomial infections admitted to different ICUs of Assiut University Hospitals (in southern Egypt) during the period from October 2009 to May 2011. The Candida isolates were from urine (55 isolates), endotracheal aspirates (42), sputum (17), blood (5), bed sores (4), oropharyngeal specimens (2) and wounds (2).

\section{Conventional methods}

Clinical specimens were processed for the isolation of Candida species according to standard procedures and were identified by conventional means (McGinnis, 1994). First cultures were obtained on Sabouraud-dextrose agar (SDA) with chloramphenicol after $48 \mathrm{~h}$ at $37^{\circ} \mathrm{C}$. All of the yeast isolates were examined by wet mount, Gram staining and germ tube (Bhavan et al., 2010), morphology and chlamydospore production on cornmeal tween 80 agar and sugar assimilation tests were done using auxanograms (Barnett et al., 2000) and KB006 HiCandida Identification Kit (HiMedia, Mumbai, India) which is a combination of
12 tests for identification of Candida species. The plastic strip has twelve wells with sterile medium for different biochemical tests as Medium for Urease detection test, and Carbohydrate Utilization Test (with eleven different sugars in respective wells as Melibiose, Lactose, Maltos, Sucrose, Galactose, Cellobiose, Inositol, Xylose, Dulcitol, Raffinose, Trehalose).

\section{Candida species identification on chromogenic media}

Separate colonies from Candida isolates on SDA were subcultured on each of the following chromogenic media: HiCrome Candida Differential agar (HiMedia, Mumbai, India), CHROMagar Candida (Paris, France), CHROMagar Candida supplemented with Pal's medium and on BIGGY Agar (HiMedia, Mumbai, India). Plates were incubated at $37^{\circ} \mathrm{C}$ for $24-48 \mathrm{~h}$. Preparation and identification of species were performed according to the manufacturer's instructions.

\section{Seminested PCR}

\section{Extraction of Candida DNA from culture}

DNA was extracted from liquid culture of Candida grown for $20-24 \mathrm{~h}$ at $30{ }^{\circ} \mathrm{C}$ in YPD (1\% yeast extract, $2 \%$ peptone, $2 \%$ dextrose) according to Harju et al. (2004). Repeated freezing-thawing of cells in a lysis buffer was used to disrupt the cell wall and release genomic DNA. Cell lysis was followed by extraction with chloroform and ethanol precipitation.

\section{PCR primers}

A 22-bp forward primer, CTSF (5-CGCATCGAT GAAGAACGCAGC-3), and a 25-bp reverse primer, CTSR (5-TCTTTTCCTCCGCTTATTGATATGC-3), capable of amplifying the 3 end of 5.8S rDNA and the 5 end of $28 \mathrm{~S}$ rDNA, including the intervening internally transcribed spacer 2 (ITS2) region, were synthesized by metabion international AG, Germany. Species-specific oligonucleotide primers for seminested PCR (snPCR) were derived from the ITS2 regions of $C$. albicans, $C$. parapsilosis, C. tropicalis, C. glabrata, C. krusei and $C$. dubliniensis and are described in Table 1 (Fujita et al., 1994; Ahmad et al., 2002; Khan et al., 2009).

\section{DNA amplification and detection}

Amplification of target DNA was carried out in thinwalled $0.2-\mathrm{mL}$ PCR tubes in a total volume of $50 \mu \mathrm{L}$ containing $1 \mu \mathrm{L}$ AmpliTaq PCR buffer I, $1 \mathrm{U}$ of AmpliTaq DNA polymerase, 10 pmol each of CTSF and CTSR primers, $1 \mu \mathrm{L}$ of DNA extracted from culture, and $0.1 \mathrm{mM}$ each deoxynucleoside triphosphate. After amplification in the first step, $1 \mu \mathrm{L}$ of the product was further amplified using the initial reverse primer (CTSR) and a species-specific forward primer in six separate tubes corresponding to each of the Candida species to be detected. For SnPCR, the reac- 
Table 1 - Primers for species identification by snPCR.

\begin{tabular}{|c|c|c|c|c|}
\hline Primer & Nucleotide sequence (5'-3') & Amplification step & Location & Amplicon size (bp) \\
\hline CADET & ATTGCTTGCGGCGGTAACGTCC & snPCR & ITS region of $C$. albicans & 105 \\
\hline CPDET & ACAAACTCCAAAАCTTCTTCCA & snPCR & ITS region of C. parapsilosis & 88 \\
\hline CTDET & AACGCTTATTTTGCTAGTGGCC & snPCR & ITS region of C. tropicalis & 106 \\
\hline CGDET & TAGGTTTTACCAACTCGGTGTT & snPCR & ITS region of $C$. glabrata & 142 \\
\hline CKDET & GGCCCGAGCGAACTAGACTTTT & snPCR & ITS region of C. krusei & 132 \\
\hline CDDET & GCTAAGGCGGTCTCTGGCGTCG & snPCR & ITS region of $C$. dubliniensis & 100 \\
\hline
\end{tabular}

tion mixture consisted of $1 \mu \mathrm{L}$ AmpliTaq PCR buffer I; $1 \mathrm{U}$ of AmpliTaq DNA polymerase; 5 pmol of CTSR together with 5 pmol of CADET, CPDET, CGDET, CTDET, CKDET, or CDDET; $1 \mu \mathrm{L}$ of the first PCR product; and $0.1 \mathrm{mM}$ each deoxynucleoside triphosphate. PCR cycling was carried out under the following conditions:

An initial denaturation step at $94{ }^{\circ} \mathrm{C}$ for $3 \mathrm{~min}$ followed by 30 cycles of denaturation at $94{ }^{\circ} \mathrm{C}$ for $1 \mathrm{~min}$, annealing at $60{ }^{\circ} \mathrm{C}$ for $30 \mathrm{~s}$, and extension at $72^{\circ} \mathrm{C}$ for $1 \mathrm{~min}$. with a final extension step at $72{ }^{\circ} \mathrm{C}$ for $10 \mathrm{~min}$. Optimum amplification was determined to be obtained with 30 cycles of the first PCR followed by 20 cycles of the snPCR according to Ahmad et al. (2002). Appropriate negative controls were included in each test run, including controls omitting the DNA template during PCR assays.

Amplified DNA fragments were separated by agarose gel electrophoresis at $80 \mathrm{~V}$ for 1 to $2 \mathrm{~h}$ on gels composed of $1 \%(\mathrm{wt} / \mathrm{vol})$ agarose (Sigma-Aldrich, Germany).The gels were exposed to UV light and photographed. The sizes of amplified DNA fragments were identified by comparison with molecular size marker DNA (100-bp DNA ladder).

\section{Statistical analysis}

Statistical analysis was performed using SPSS software version 16 (SPSS Inc., Chicago, USA). Values are presented as percentages of the group from which they were derived. Sensitivities and specificities of chromogenic media for the species were calculated as follows:

$$
\begin{aligned}
& \text { Sensitivity }=\frac{\text { true positive }}{(\text { true positive }+ \text { false negative })} \times 100 \\
& \text { Sensitivity }=\frac{\text { true negative }}{(\text { true negative }+ \text { false positive })} \times 100
\end{aligned}
$$

Percent agreement was determined by the number of isolates positive by conventional methods /number of PCR-positive isolates x 100 .

\section{Results}

\section{Candida identification by conventional methods}

Among the 127 Candida isolates, the majority were non-albicans $(95 / 127,75 \%)$. C. tropicalis was the most frequent ( $46.5 \%$ or 59 strains) followed by C. glabrata
( $17.4 \%$ or 22 stains). On the other hand, C. albicans constituted $25 \%$ of the isolates $(32 / 127)$. The remaining species constituted much lower percentages as shown in Figure 1.

\section{Candida identification on four chromogenic media}

By culture on four chromogenic media, different isolated Candida spp. showed different colony colors and morphology which are summarized in Table 2 .

The sensitivities and specificities of the chromogenic media in comparison to the conventional methods are shown in Table 3. HiCrome Candida and CHROMagar Candida media had comparably high sensitivities and specificities in identification of C. albicans, C. tropicalis , C. glabrata and C. krusei. CHROMagar Candida was the only medium that identified C. parapsilosis with a high sensitivity and specificity. BiGGY agar showed the lowest sensitivity and specificity in the identification of $C$. albicans, C. tropicalis and C. glabrata. CHROMagar Candida medium supplemented by Pal's medium was the only chromogenic medium that detected $C$. dubliniensis with $100 \%$ sensitivity and specificity.

\section{PCR results}

Out of the 127 Candida isolates, only 123 isolates were tested by PCR. C. rugosa (2 isolates) and $C$. haemulonii (1 isolate) were not tested. Regarding PCR am-

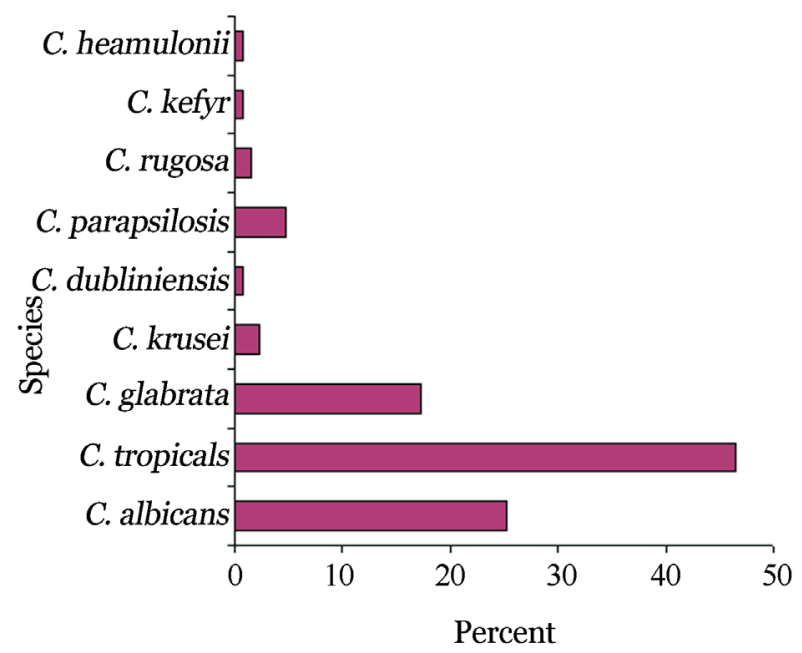

Figure 1 - Frequency of different Candida species identified by conventional methods. 


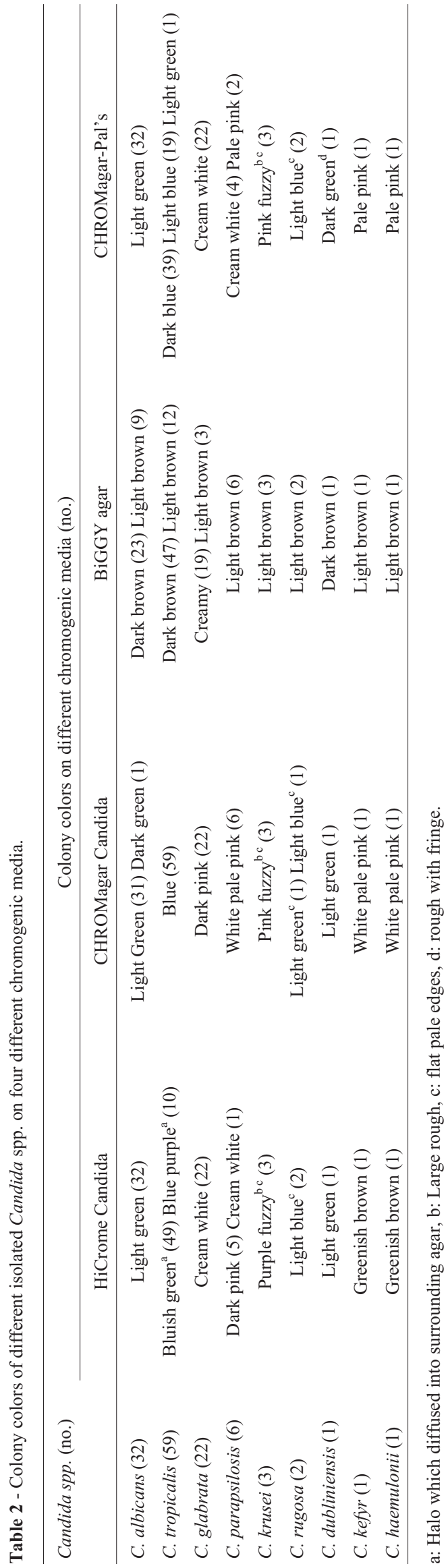

plification of Candida rDNA with universal fungal primers, the first round of PCR amplification of rDNA was performed with genomic DNA from different Candida spp. and non-fungal organisms with CTSF and CTSR primers and the amplified products were detected by agarose gel electrophoresis (AGE). The PCR amplification with these two primers and genomic DNA from $C$. albicans, $C$. tropicalis, C. glabrata, C.krusei, C. dubliniensis, and $C$. parapsilosis resulted in amplification of DNA fragments of $344,334,423,335,337$ and 317 bp respectively (Figure 2).

In sn PCR, reamplification of the product of the first PCR with CTSR and the species-specific primers corresponding to the ITS2 sequences from $C$. albicans, $C$. tropicalis, C. glabrata, C. krusei, C. dubliniensis, and C. parapsilosis resulted in specific amplification of single DNA products of the expected sizes $(105,106,142,132$, 100,88 bp respectively) (Figure 3 ) .

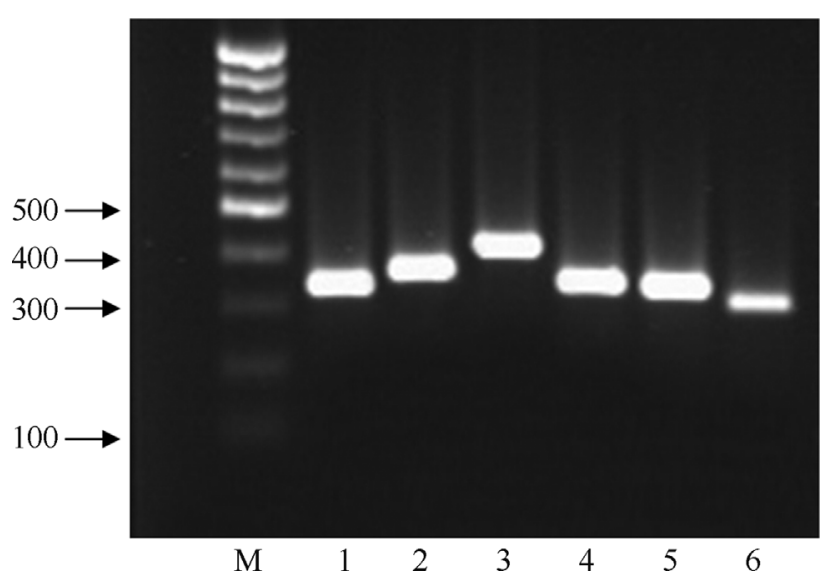

Figure 2 - PCR amplification of genomic DNAs of 6 Candida spp. with universal fungal primers. Lane M: 100-bp molecular size marker, Lane 1: C. tropicalis, Lane 2: C. albicans, Lane 3: C. glabrata, Lane 4: C. krusei, Lane 5: C. dubliniensis, and Lane 6: C. parapsilosis.

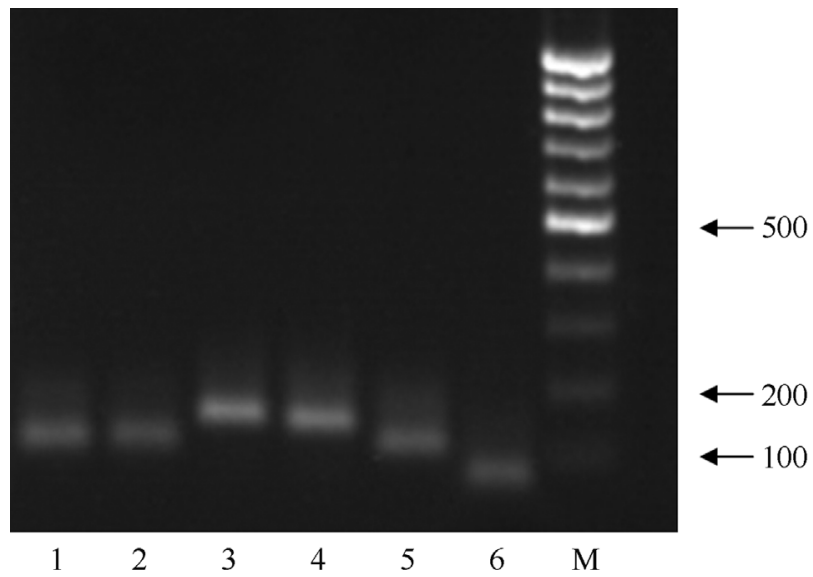

Figure 3 - Lanes 1 to 6, snPCR amplification of DNAs from C. tropicalis, C. albicans, C. glabrata, C. krusei, C. dubliniensis and C. parapsilosis respectively, using primer CTSR with primers CTDET, CADET, CGDET, CKDET, CDDET, and CPDET respectively. Lane M, 100-bp molecular size marker. 
Table 3 - Sensitivity and specificity of the chromogenic media in relation to the conventional phenotypic methods.

\begin{tabular}{|c|c|c|c|c|c|c|c|c|}
\hline \multirow[t]{2}{*}{ Candida spp. (no) } & \multicolumn{2}{|c|}{ HiCrome Candida } & \multicolumn{2}{|c|}{ CHROMagar Candida } & \multicolumn{2}{|c|}{ BiGGY agar } & \multicolumn{2}{|c|}{ CHROMagar-Pal's } \\
\hline & Sensitivity & Specificity & Sensitivity & Specificity & Sensitivity & Specificity & Sensitivity & Specificity \\
\hline C. albicans (32) & 100 & 98.9 & 96.9 & 97.9 & 71.9 & 50.5 & 100 & 100 \\
\hline C. tropicalis (59) & 100 & 100 & 100 & 98.5 & 79.7 & 66.1 & 100 & 97 \\
\hline C. glabrata (22) & 100 & 99 & 100 & 100 & 86.4 & 100 & - & - \\
\hline C. parapsilosis (6) & - & - & 100 & 98.3 & - & - & - & - \\
\hline C. krusei (3) & 100 & 100 & 100 & 100 & - & - & 100 & 100 \\
\hline C. rugosa (2) & - & - & - & - & - & - & - & - \\
\hline C. dubliniensis (1) & - & - & - & - & - & - & 100 & 100 \\
\hline
\end{tabular}

The results of snPCR in species identification were concordant with those of the conventional phenotypic methods for all isolates of $C$. albicans, C. tropicalis, $C$. glabrata, C. krusei, and C. dubliniensis species. But for the 3 isolates of $C$. parapsilosis, there was discrepancy between SnPCR and the phenotypic tests. Thus, the overall concordance of the snPCR compared to the conventional phenotypic tests in the identification of Candida species was found to be $97.5 \%$ (120 of 123) as shown in Table 4.

\section{Discussion}

Rapid presumptive identification of yeast species is quite difficult in resource- limited countries due to the lack of training, proper reagents and equipments. In order to reduce the financial burden of the poor patients, these laboratories do not go beyond the germ tube test and limit their diagnosis to C. albicans or NAC. The biochemical assimilation and fermentation tests are not used in these laboratories due to lack of resources, expertise and time required for these tests which increase the cost of mycology cultures (Pfaller et al., 1996). As a result, direct selection of appropriate agents for antifungal therapy or prophylaxis becomes almost impossible. That's why there is always a need for media which help not only in the isolation but also in the identification at the species level (Nadeem et al., 2010).

Table 4 - Agreement between the conventional phenotypic methods and sn PCR in the identification of Candida species.

\begin{tabular}{lccc}
\hline Candida spp. & \multicolumn{2}{c}{ No. of isolates identified by: } & \% agreement \\
\cline { 2 - 3 } & $\begin{array}{c}\text { Conventional } \\
\text { phenotypic methods }\end{array}$ & snPCR & \\
\hline C. albicans & 32 & 32 & 100 \\
C. tropicalis & 59 & 59 & 100 \\
C. glabrata & 22 & 22 & 100 \\
C. parapsilosis & 6 & 3 & 50 \\
C. krusei & 3 & 3 & 100 \\
C. dubliniensis & 1 & 1 & 100 \\
Total & 123 & 120 & 97.5 \\
\hline
\end{tabular}

Chromogenic media have the advantage of rapid identification of Candida species, technically simple preparation (by boiling), rapid and cost effective compared to technically demanding time consuming and expensive conventional method (Vijaya et al., 2011). In this study, the colony appearance of 127 Candida isolates on four chromogenic media was compared in order to identify the most easy and reliable one for rapid identification of different Candida species. Compared to the results of the conventional methods, the sensitivity and specificity of HiCrome Candida Differential agar (HiMedia, Mumbai, India) were determined as $100 \%$ and $98.9 \%$ for C. albicans, $100 \%$ and $100 \%$ for C. tropicalis, $100 \%$ and $99 \%$ for $C$. glabrata, and $100 \%$ and $100 \%$ for C. krusei.

Regarding $C$ albicans, our results are comparable to many previous studies. Willinger et al. (2001) reported $98.8 \%$ sensitivity and $100 \%$ specificity. Baradkar et al. (2010) reported a sensitivity and specificity of HiChrom Candida agar to be 96.55 and $96.42 \%$ respectively. Yucesoy and Marol (2003)reported the sensitivity and specificity to be $99.4 \%$ and $100 \%$ respectively.

For C. tropicalis, similar results were previously reported by Yucesoy and Marol (2003). Baradkar et al. (2010) found the sensitivity and specificity to be $100 \%$. On the other hand, Peng et al. (2007) showed 100\% sensitivity and $78.8 \%$ specificity.

We couldn't distinguish $C$. dubliniensis from $C$. albicans isolates as we had only one isolate that gave light green colored growth so it is difficult to evaluate HiChrom Candida agar for the identification of this species.

For C. parapsilosis, $83 \%$ of the isolates produced pink colonies while $17 \%$ produced cream colonies similar to those of C. glabrata. As C. glabrata doesn't produce pseudohyphae on corn meal/Tween 80 agar, combination of the two media can distinguish the two species within $48 \mathrm{~h}$ as compared to $48 \mathrm{~h}$ to 2 weeks required for sugar assimilation tests (Willinger et al., 2001). These results were similar to those reported by Girgis et al. (2009) and Vijaya et al. (2011). We reported a sensitivity and specificity for identification of C. glabrata to be $100 \%$ and $99 \%$ respectively comparable to Willinger et al. (2001) but higher than other studies (Yucesoy and Marol, 2003; Peng et al., 2007; 
Baradkar et al., 2010). The results in this study concerning C. parapsilosis differed from Baradkar et al. (2010) who described that the majority of growth gave cream colored colonies $(8 / 10)$ while the minority $(2 / 10)$ produced pink colonies. C. rugosa produced characteristic easily identifiable colony color and morphology on HiCrome Candida Differential agar. Other Candida species (C. kefyr and $C$. haemulonii) could not be differentiated from each other on this medium as both produced greenish brown colonies. Unfortunately, we couldn't find any other reports evaluating HiCrome Candida Differential agar (HiMedia, Mumbai, India) for identification of these less common Candida species.

The sensitivity and specificity of CHROMagar Candida (Paris, France) were determined as $96.9 \%$ and $97.9 \%$ for C. albicans, $100 \%$ and $98.5 \%$ for C. tropicalis, $100 \%$ and $100 \%$ for C. glabrata, $100 \%$ and $100 \%$ for $C$. krusei, and $100 \%$ and $98.3 \%$ for C. parapsilosis respectively. C. rugosa appears to typically produce a readily identifiable and unique color/colony type on CHROMAgar Candida as previously reported (Hospenthal et al., 2006). In agreement, Nadeem et al. (2010) reported that CHROMAgar Candida correctly identified the majority of C. albicans, C. tropicalis, C. glabrata, and C. krusei with comparable high sensitivities and specificities.

Regarding C. glabrata, we didn't agree with Hospenthal et al. (2006), who reported that they couldn't distinguish $C$. glabrata from others that gave pink to lavender coloured colonies. C. kefyr and C. haemulonii could not be differentiated from $C$. parapsilosis on CHROMagar Candida. Also, C. dubliniensis could not be distinguished from C. albicans green colonies. These results were consistent with those of Hospenthal et al. (2006) and Nadeem et al. (2010).

The addition of Pal's agar to CROMagar Candida medium facilitated the differentiation of $C$. dubliniensis from $C$. albicans with $100 \%$ sensitivity and specificity for both species, where C. albicans isolates appeared as light green smooth colonies while $C$. dubliniensis showed distinguishable dark green rough colonies which were accompanied by the production of chlamydospores. These results were in accordance with those of Sahand et al. (2005). Other Candida species that were assayed in this study showed rough or smooth colonies in CHROMagar Candida supplemented with Pal's agar; however, they never developed green colonies nor produced chlamydospores, making their misidentification as $C$. albicans or $C$. dubliniensis highly unlikely. Interestingly, C. tropicalis and C. krusei retained a distinguishable blue and pink-and-rough colony aspect, respectively, that allowed their easy identification on CHROMagar Candida medium supplemented with Pal's agar with sensitivity and specificity detected as $100 \%$ and $97 \%$ for C. tropicalis and $100 \%$ for C. krusei respectively. On the other hand, C. glabrata, C. parapsilosis, C. kefyr and $C$. haemulonii produced indistinguishable pale pink to cream white smooth colonies making it difficult to identify them on CHROMagar Candida supplemented with Pal's medium. These results were in harmony with Sahand et al. (2005).

The sensitivity and specificity of BiGGY agar were detected as $71.9 \%$ and $50.5 \%$ for C. albicans, $79.7 \%$ and $66.1 \%$ for C. tropicalis, and $86.4 \%$ and $100 \%$ for $C$. glabrata respectively. It was difficult to differentiate the colors of $C$. albicans and $C$. tropicalis colonies on this medium. In the present study, most $C$. glabrata strains $(86.4 \%$, 19/22) showed distinctive appearance on BiGGY agar after $48 \mathrm{~h}$. Brown color was only observed at the first streaks of the cultures, where the colonies were very crowded, while other areas especially single colonies were very small and creamy colored. These data agreed with those of Yücesoy and Marol (2003) with the exception of C. krusei which they reported to show typical distinctive appearance on BiGGY agar with $100 \%$ sensitivity and specificity. The discrepancy may be due to the small number of C. krusei ( 3 isolates) tested in our study compared to 12 isolates in the study of Yücesoy and Marol (2003). The lower sensitivity and specificity of BiGGY agar to identify commonly isolated Candida species potentially limits the clinical usefulness of this agar despite its low cost compared to other chromogenic media.

As Culture-based phenotypic identification techniques are slow and especially prone to misidentification of fungal pathogens. Numerous DNA-based methods have developed to diagnose Candida infections. In the present study, snPCR assays targeting species-specific sequences in the rDNA have been used for the specific detection of six clinically important Candida species. We used the sn PCR amplification method according to many previous studies (Ahmad et al., 2002, 2004; Çerikçioglu et al., 2010). The reamplification step introduced has resulted in maximum sensitivity of the assay with a specificity of $100 \%$ (Ahmad et al., 2004; Çerikçioglu et al., 2010).

The target for snPCR amplification was rDNA which is considered to be most suitable for sensitive detection of Candida because it is present in multiple copies (50 to 100 copies) per Candida genome (Reiss et al., 1998). Moreover, between the highly conserved rDNA subunits are the internally transcribed spacers, which contain sequences unique to each Candida species, and thus the use of primers corresponding to these regions facilitates species identification (Ahmed et al., 2002).

The results presented in this study showed a $97.5 \%$ overall concordance of the snPCR with the conventional methods including the use of CHROMagar Candida medium in the identification of Candida species which were comparable to those of Ahmad et al. (2002) and Girgis et al. (2009). Sahiner et al. (2011) reported that the traditional phenotypic methods including CHROMagar Candida medium yielded the same results (100\% compatible) as PCR in the species identification of Candida isolates. 
The discrepancy we found was among 3 isolates which were phenotypically identified as $C$. parapsilosis but they showed negative results when tested with all species-specific primers used in the study (50\%). Although the precise reason for this discrepancy remains unclear, it could be related to inadequacy of the presently available phenotypic Candida identification methods. Further confirmation by DNA sequencing may be required to correctly identify these isolates. Ahmad et al. (2004) reported a closely related finding. They found that no amplification was detected in snPCR from C. krusei, C. lusitaniae and $C$. dubliniensis.

The snPCR used in this study has an average processing time of 9 to $10 \mathrm{~h}$, does not require the use of hybridization probes and radioactive substances, and is specific. The snPCR appears to be at least 100 times more sensitive than the multiplex PCRs (Ahmed et al., 2002).

In conclusion, CHROMAgar Candida medium (CHROMagar Company, Paris, France) is an easy and accurate method for presumptive identification of the most commonly encountered Candida spp. (C. albicans, C. tropicalis, C. glabrata, C. parapsilosis and C. krusei) within $48 \mathrm{~h}$. Although sn PCR identified the species within $8-10 \mathrm{~h}$ but its cost-effectiveness should also be considered. So these phenotypic methods should be considered in the identification of Candida species in areas with limited resources.

\section{References}

Ahmad S, Khan Z, Mustafa AS, Khan ZU (2002) Seminested PCR for diagnosis of candidemia: comparison with culture, antigen detection, and biochemical methods for species identification.. J Clin. Microbiol. 40(7):2483-2490.

Ahmad S, Mustafa AS, Khan Z, Al-Rifaiy AI, Khan ZU (2004) PCR-enzyme immunoassay of rDNA in the diagnosis of candidemia and comparison with amplicon detection by agarose gel electrophoresis. Int. J Med Microbiol 294:45-51.

Baradkar VP, Mathur M, Kumar S (2010) Hichrom Candida agar for identification of Candida species. Indian. J. Pathol. Microbiol 53(1):93-94.

Barnett JA, Pane RW, Yarrow D (2000) Yeasts: Characteristics and Identification. Cambridge University Press: Cambridge, 3rd edition, pp 26-27.

Bernal S, Mazuelos ME, Garcia M, Aller AI, Martinez MA, Gutierrez MJ (1996) Evaluation of CHROMagar Candida medium for the isolation and presumptive identification of species of Candida of clinical importance Diagn Microbiol Infect Dis 24(4):201-204.

Bhavan PS, Rajkumar R, Radhakrishnan S, Seenivasan C, Kannan S (2010) Culture and Identification of Candida albicans from Vaginal Ulcer and Separation of Enolase on SDS-PAGE. Interna J Biol 2:84-94.

Çerikçioglu N, Aksu B, Dal, TD, Deniz U, Bilgen HS, Özek E, Söyletir G (2010) Seminested PCR for detection and identification of Candida species directly from blood culture bottles. New. Microbiologica. 33:57-62.
Fluit AC, Jones ME, Schmitz FJ, Acar J, Gupta R, Verhoef J (2000) Antimicrobial susceptibility and frequency of occurrence of clinical blood isolates in Europe from the SENTRY antimicrobial surveillance program, 1997 and 1998. Clin Infect Dis 30(3):454-460.

Fujita S, Lasker BA, Lott TJ, Reiss E, Morrison CJ (1995) Microtitration plate enzyme immunoassay to detect PCR-amplified DNA from Candida species in blood. J Clin Microbiol 33:962-967.

Girgis SA, El-Mehalawy AA, Rady LM (2009) Comparison between culture and non-culture based methods for detection of Nosocomial fungal infections of Candida spp. in intensive care unit patients. Egypt. Acad. J. Biolog. Sci. 1:37-47.

Harju S, Fedosyuk H, Peterson KR (2004) Rapid isolation of yeast genomic DNA: Bust n' Grab. BMC Biotechnol 4:8.

Hospenthal DR, Beckius ML, Floyd KL, Horvath LL, Murray CK (2006) Presumptive identification of Candida species other than C. albicans, C. krusei, and C. tropicalis with the chromogenic medium CHROMagar Candida. Ann Clin Microbiol Antimicrobiol 3:5-1.

Hospenthal DR, Murray CK, Rinaldi MG (2004) The role of antifungal susceptibility testing in the therapy of candidiasis. Diagn Microbiol Infect Dis 48(3):153-160.

Khan Z, Mustafa AS, Alam FF (2009) Real-time LightCycler polymerase chain reaction and melting temperature analysis for identification of clinically important Candida spp. J Microbiol Immunol Infect 42:290-295.

Liguori G, Lucariello A, Colella G, De Luca A, Marinelli P (2007) Rapid identification of Candida species in oral rinse solutions by PCR. J Clin Pathol 60:1035-1039.

McGinnis MR (1994) Mycology. In Isenberg H. D. (ed.). Clinical microbiology procedure handbook. American Society for Microbiology, Washington D.C., pp 6.1-6.12.

Murray CK, Beckius ML, Green JA, Hospenthal DR (2005) Use of chromogenic medium for the isolation of yeasts from clinical specimens. J Med Microbiol 54:981-985.

Nadeem, S.G.; Hakim, S.T.; Kazmi, S.U. (2010). Use of CHROMagar Candida for the presumptive identification of Candida species directly from clinical specimens in resource-limited settings. Libyan J Med. 5:2144-2150.

Nguyen MH, Peacock JE, Morris A J, Tanner DC, Nguyen ML, Snydman DR, Waqener MM, Rinaldi MG, Yu VL (1996) The changing face of candidemia: emergence of nonCandida albicans species and antifungal resistance. Am. J Med 100(6):617-623.

Peng, C.F.; Lee, K.M.; Lee, S.H. (2007). Characterization of two chromogenic media of Candida $\mathrm{ID}_{2}$ and CHROMagar Candida for preliminary identification of yeasts. J Biomed Lab Sci 19:63-68.

Pfaller MA, Houston A, Coffmann S (1996) Application of CHROMagar Candida for rapid screening of clinical specimens for Candida albicans, Candida tropicalis, Candida krusei, and Candida (Torulopsis) glabrata. J Clin Microbiol 34(1):58-61.

Reiss E, Tanaka K, Bruker G, Chazalet V, Coleman D, Debeaupuis JP, Hanazawa R, Latge JP, Lortholary J, Makimura K, Morrison CJ, Murayama SY, Naoe S, Paris S, Sarfati J, Shibuya K, Sullivan D, Uchida K, Yamaguchi H (1998) Molecular diagnosis and epidemiology of fungal infections. Med Mycol 36:249-256. 
Sahand IH, Moragues MD, Eraso E, Villar-Vidal M, Quindos G, Ponton J (2005). Supplementation of CHROMagar Candida medium with Pal's medium for rapid identification of Candida dubliniensis. J Clin Microbiol 43 (11):5768-5770.

Sahiner F, Ergünay K, Ozyurt M, Ardyç N, Hosbul T, Haznedaroglu T (2011) Phenotypic and genotypic identification of Candida strains isolated as nosocomial pathogens. Mikrobiyol Bul 45:478-488.

Vijaya D, Harsha TR, Nagaratnamma T (2011) Candida Speciation Using Chromagar. J Clin Diagn Res 5:755-757.

Wahyuningsih R, Freisleben HJ, Sonntag HG, Schnitzler P (2000) Simple and rapid detection of Candida albicans DNA in se- rum by PCR for diagnosis of invasive candidiasis. $J$ Clin Microbiol 38:3016-3021.

Willinger B, Hillowoth C, Selitsch B, Manafi M (2001) Performance of Candida ID, a new chromogenic medium for presumptive identification of Candida species, in comparison to CHROMagar Candida. J Clin Microbiol 39(10):3793-3795.

Yucesoy M, Marol S (2003) Performance of CHROMagar Candida and BIGGY agar for identification of yeast species. Ann Clin Microbiol Antimicrobiol 2:1-8.

All the content of the journal, except where otherwise noted, is licensed under a Creative Commons License CC BY-NC. 